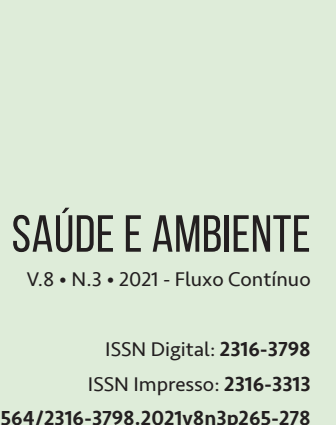

DOI: 10.17564/2316-3798.2021v8n3p265-278

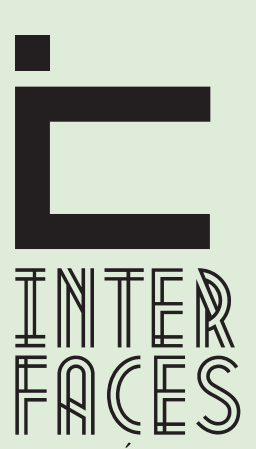

CIENTÍFICAS

\title{
RESTAURANTES DE INSTITUIÇÕES PÚBLICAS DE ENSINO: UM OLHAR SOBRE DESEMPENHO AMBIENTAL E CONDICŌ̃ES HIGIENICOSSANITÁRIAS
}

RESTAURANTS OF PUBLIC EDUCATION INSTITUTIONS: A LOOK AT ENVIRONMENTAL PERFORMANCE AND HYGIENIC-SANTTARY CONDTIONS

RESTAURANTES DE INSTITUCIONES DE EDUCACIÓN PÚBLICA: UNA MIRADA AL DESEMPEÑO AMBIENTAL Y LAS CONDICIONES HIGIÉNICO-SANITARIAS

Josimara Pereira Nogueira ${ }^{1}$

Maria Hatjiathanassiadou ${ }^{2}$

Virgílio José Strasburg ${ }^{3}$

Priscilla Moura Rolim ${ }^{4}$

Larissa Mont'Alverne Jucá Seabra ${ }^{5}$

\section{RESUMO}

Os serviços de alimentação institucionais devem garantir o fornecimento de uma alimentação segura e de qualidade para os usuários e ao mesmo tempo ser ambientalmente responsável com o uso dos recursos naturais. Este estudo teve como objetivo avaliar o desempenho ambiental e as condições higienicossanitárias relacionados à produção de refeições em Restaurantes Institucionais (RI) de ensino público em um estado do nordeste brasileiro. A pesquisa caracterizou-se como descritiva exploratória de natureza transversal/observacional, realizada em seis restaurantes de instituições de ensino público, sendo 3 restaurantes universitários e 3 restaurantes de institutos de ensino médio técnico. 0 desempenho ambiental foi avaliado por meio de um instrumento que abordava o uso de água, energia elétrica, gás, produtos químicos e gestão de resíduos. Para a avaliação das condições higienicossanitárias nos RI foi utilizada uma lista de verificação contemplando instalações, processos, documentação e registros referente às boas práticas de manipulação dos alimentos. Os resultados mostraram que, em relação ao desempenho ambiental, todos os RI alcançaram um escore percentual classificado como bom ou muito bom (60,5 a 86,5\%). Gestão de resíduos e uso de energia elétrica obtiveram respectivamente o melhor e pior percentual médio de adequação. Em relação às condições higienicossanitárias, a média de adequação geral dos IR variou entre $47,3 \%$ a 91,7\%, sendo o item documentação e registro o que obteve menor adequação em todos os restaurantes.

\section{PALAVRAS-CHAVE}

Alimentação Coletiva. Serviços de Alimentação. Segurança e Qualidade dos Alimentos. 


\section{ABSTRACT}

Institutional food services must guarantee the provision of safe and quality food for users and at the same time be environmentally responsible with the use of natural resources. This study aimed to evaluate the environmental performance and the hygienic-sanitary conditions related to the production of meals in Institutional Restaurants (IR) of public education in a state of northeastern Brazil. The research was characterized as exploratory descriptive of a transversal/observational nature, performed in six restaurants of public education institutions, 3 university restaurants and 3 restaurants of technical high school institutes. Environmental performance was assessed using an instrument that addressed the use of water, electricity, gas, chemicals and waste management. For the evaluation of hygienic-sanitary conditions in the IR, a checklist was used, including facilities, processes, documentation and records regarding good food handling practices. The results showed that, in relation to environmental performance, all IR reached a percentage score classified as good or very good (60.5 to $86.5 \%$ ). Waste management and use of electric energy obtained the best and worst average percentage of adequacy, respectively. In relation to hygienic-sanitary conditions, the average of the general adequacy of the IRs ranged from $47.3 \%$ to $91.7 \%$, with the item documentation and registration having the lowest adequacy in all restaurants.

\section{KEYWORDS}

Collective Feeding. Food Services. Food quality.

\section{RESUMEN}

Los servicios alimentarios institucionales deben garantizar la provisión de alimentos inocuos y de calidad para los usuarios y al mismo tiempo ser ambientalmente responsables con el uso de los recursos naturales. Este estudio tuvo como objetivo evaluar el desempeño ambiental y las condiciones higiénico-sanitarias relacionadas con la producción de comidas en Restaurantes Institucionales (RI) de educación pública en un estado del noreste de Brasil. La investigación se caracterizó por ser descriptiva exploratoria de carácter transversal/observacional, realizada en seis restaurantes de instituciones de educación pública, siendo 3 restaurantes universitarios y 3 restaurantes de institutos técnicos. El desempeño ambiental se evaluó mediante un instrumento que abordó el uso del agua, electricidad, gas, químicos y manejo de desechos. Para la evaluación de las condiciones higiénico-sanitarias en el RI, se utilizó una lista de verificación, que incluye instalaciones, procesos, documentación y registros sobre buenas prácticas de manipulación de alimentos. Los resultados mostraron que, en relación al desempeño ambiental, todos los RI alcanzaron un puntaje porcentual clasificado como bueno o muy bueno (60,5 hasta $86,5 \%)$. La gestión de residuos y el uso de energía eléctrica 
obtuvieron el mejor y el peor porcentaje medio de adecuación, respectivamente. En cuanto a las condiciones higiénico-sanitarias, el promedio de adecuación general del RI osciló entre el 47,3\% y el $91,7 \%$, siendo la documentación y registro del ítem el menos adecuado en todos los restaurantes.

\section{PALABRAS CLAVE}

Alimentación Colectiva; Servicios de Alimentación; Seguridad y calidad de los alimentos.

\section{INTRODUÇ̧̃̃O}

Devido ao modo de vida atual e a crescente necessidade de realizar parte das refeições fora de casa, os serviços de alimentação têm ganhado cada vez mais espaço na vida das pessoas (SANTOS et al., 2011). Diante desse contexto, esses serviços, também denominados de Unidades de Alimentação e Nutrição (UAN), desempenham um papel fundamental na sociedade atual, não somente no quesito saúde, mas também nas esferas social, econômica e ambiental (CFN, 2018; NOGUEIRA et al., 2020).

As UAN têm como objetivo fornecer uma alimentação saudável, nutricionalmente adequada, que atenda aos padrões higienicossanitários e que seja compatível com as condições financeiras do serviço (BRASIL, 2004; 2014). De acordo com Associação Brasileira de Empresas de Refeições Coletivas (ABERC), estima-se que o setor no ano de 2020 ofereceu em média 22,33 milhões de refeições por dia, faturando cerca de R\$37,21 bilhões de reais e empregando aproximadamente 250 mil trabalhadores (ABERC, 2021).

É importante destacar que, sabendo que promover a alimentação saudável é um dos objetivos da UAN, por um tempo o conceito de "alimentação saudável” estava restrito a composição química dos alimentos e oferta dietética adequada. No entanto, essa visão limitada exclui todo o aspecto sistêmico da alimentação, onde se considera todo o ciclo de vida, desde a produção até a distribuição e consumo, que geram impactos ambientais e econômicos e devem ser considerados pelos locais de produção de refeições (HARMON et al., 2007).

Frente a essas questões, na década de 1980 foi formulado o conceito de "Nutrição Sustentável" referindo-se a uma alimentação saudável que abrangem os aspectos de saúde, sociedade, economia, cultura, bem como o meio ambiente; além de levar em consideração todos os estágios que compõe a cadeia de produção dos alimentos para que uma alimentação seja considerada sustentável (VON KOERBER et al., 2017).

Trazer o conceito de nutrição sustentável e dar ênfase as dimensões ambiental e de saúde são imprescindíveis nos espaços que operam na produção de refeições para a coletividade, uma vez que o uso racional de recursos naturais, como recursos hídricos e energéticos, o uso da terra, a redução da geração de resíduos, além da oferta de uma alimentação segura, colaboram para produção e consumo responsável - convergindo com os objetivos de Desenvolvimento Sustentável da Agenda 2030 (HARMON et al., 2007; UN, 2015; WALLS et al., 2019).

O Desempenho Ambiental (DA) pode ser caracterizado como os resultados mensuráveis da gestão de uma organização sobre seus aspectos ambientais investigados (ABNT, 2015). Desse modo, deter- 
minar o DA da produção de refeições nas UAN se faz necessário por considerar sua relevância em pontuar as atividades/aspectos que geram danos ao meio ambiente (STRASBURG, 2016).

No contexto da nutrição sustentável, concomitantemente à interface ambiental, cabe mencionar que refeições elaboradas sem o padrão higienicossanitário adequado, agregam riscos eminentes à saúde dos comensais e desperdícios gerados pela falta de controle do processo produtivo. Adicionalmente, considerando o atual cenário pandêmico e/ou pós-pandêmico de COVID-19 (Sars-CoV-2), inúmeros são os desafios para produção de refeições coletivas, assim o cumprimento da legislação sanitária vigente deve ser ainda mais discutido e implementados nos serviços de alimentação (BEZERRA, 2020; BRASIL, 2004; CFN, 2020; NOGUEIRA et al., 2019; SILVA JÚNIOR, 2007).

Portanto, é inegável que os espaços de alimentação coletiva possuem responsabilidades complexas que envolvem além da qualidade nutricional do cardápio, questões socioambientais. Desta forma, este estudo teve por objetivo avaliar o desempenho ambiental e as condições higienicossanitárias relacionados à produção de refeições em Restaurantes Institucionais (RI) de ensino público em um estado do nordeste brasileiro.

\section{MÉTODOS}

O estudo foi do tipo descritivo exploratório de natureza transversal/observacional, realizado em restaurantes de instituições de ensino público federal. A partir de uma amostragem não-probabilística por conveniência, foram selecionados três restaurantes universitários e três restaurantes de institutos federais de ensino, denominados nesta pesquisa de R1 a R6, localizados em Natal e região metropolitana, Rio Grande do Norte.

A coleta dos dados ocorreu no ano de 2019, sendo realizada quatro visitas com intervalo não superior a sete dias em cada restaurante. 0 estudo obteve aprovação do Comitê de Ética em Pesquisa do Hospital Universitário Onofre Lopes da Universidade Federal do Rio Grande do Norte (UFRN), sob o parecer de número 2.989 .483$.

\subsection{AVALIAÇ̃̃O DO DESEMPENHO AMBIENTAL}

Para avaliação do Desempenho Ambiental (DA) das atividades relacionadas a produção de refeições nos restaurantes institucionais, foi aplicada uma lista de "Verificação de Aspectos e Impacto Ambiental para Serviços de Alimentação e Nutrição” desenvolvida por Strasburg (2016). Em cada local, foi aplicada em uma única vez esse instrumento para levantamento dos dados.

Os dados foram coletados por pesquisadores previamente treinados para familiarização com o instrumento. $\mathrm{Na}$ impossibilidade de avaliação por contato visual, foi requerida a participação do responsável técnico e, quando necessário, solicitado a documentação de comprovação para qualquer esclarecimento durante o momento da coleta.

O instrumento de avaliação apresenta um total de 56 itens distribuídos em cinco parâmetros distintos, sendo estes: 1. Água (11 itens); 2. Energia Elétrica (12 itens); 3. Gás (oito itens); 4. Produtos Químicos (10 itens); e 5. Gestão de Resíduos (15 itens). Cada parâmetro foi analisado, considerando 
os itens da ferramenta de verificação e sua aplicação à realidade de cada local, sendo assim, calculou-se o percentual de adequação por parâmetro (STRASBURG, 2016).

Por fim, foi calculada uma média de adequação geral para cada restaurante institucional, sendo obtido um diagnóstico do DA relacionado aos aspectos e impacto gerados pelas atividades de cada serviço. Os critérios de classificação são definidos da seguinte forma: até $50,0 \%$ de adequação, desempenho ambiental "insatisfatório"; de 51,0\% a 75,0\% "bom" desempenho ambiental e de 76,0\% a 100,0\% desempenho ambiental "muito bom” (STRASBURG, 2016).

\subsection{AVALIAÇ̃̃O DAS CONDIÇÕES HIGIENCIOSSANITÁRIAS}

Com a finalidade de avaliar as condições higienicossanitárias de produção da alimentação nos restaurantes em estudo, foi aplicada uma lista de verificação referente às boas práticas de manipulação dos alimentos. 0 instrumento utilizado foi desenvolvido por Saccol e colaboradores (2012). Esta lista de verificação é composta por 12 blocos de avaliação, está organizada e estruturada de acordo com a Resolução da Diretoria Colegiada (RDC) no 216/2004 da Agência Nacional de Vigilância Sanitária (ANVISA) (BRASIL, 2004).

A lista foi aplicada em triplicata, em três dias distintos, com intervalo não superior a quinze dias em cada local. Cada item pôde ser classificado como adequado (AD); inadequado (IN) ou não se aplica (NA) ao local estudado. A opção "NA" foi enquadrada ao item que não opera na realidade do serviço (BRASIL, 2004; SACCOL et al., 2012). A análise dos dados resultou em um diagnóstico das condições de produção de refeições dos locais, fundamentado no cumprimento dos requisitos legais, sendo classificado como: "Muito ruim": quando atendeu de 0,0\% a 19,0\%; “Ruim": de 20,0\% a 49,0\%; "Regular": de 50,0\% a 69,0\%; “Boa”: de 70,0\% a 90,0\% e "Muito boa”: de 91,0\% a 100,0\%. Para esta pesquisa o valor de adequação $\geq 70,0 \%$ foi estabelecido como "ponto de corte" para classificar os serviços como "adequado" no que se refere às condições higienicossanitárias (SACCOL et al., 2012).

\subsection{ANÁLISE DOS DADOS}

Os dados obtidos por meio desses instrumentos de avaliação foram tabulados e processados no software Microsoft Office Excel ${ }^{\varpi}$ (versão 15.0), onde foi calculada frequência absoluta e relativa, percentuais, e aplicada estatística descritiva (média e desvio padrão).

\section{RESULTADOS}

\subsection{DESEMPENHO AMBIENTAL}

Os resultados da avaliação do desempenho ambiental nos restaurantes institucionais (RI) estão descritos na Figura 1. Identificou-se que a maioria dos RI apresentaram desempenho considerado "Bom” para os aspectos avaliados, e que dois dos RI alcançaram classificação como "Muito Bom”. 
Figura 1 - Média de adequação geral de Desempenho Ambiental para os seis Restaurantes Institucionais (RI) de ensino do Rio Grande do Norte, 2019

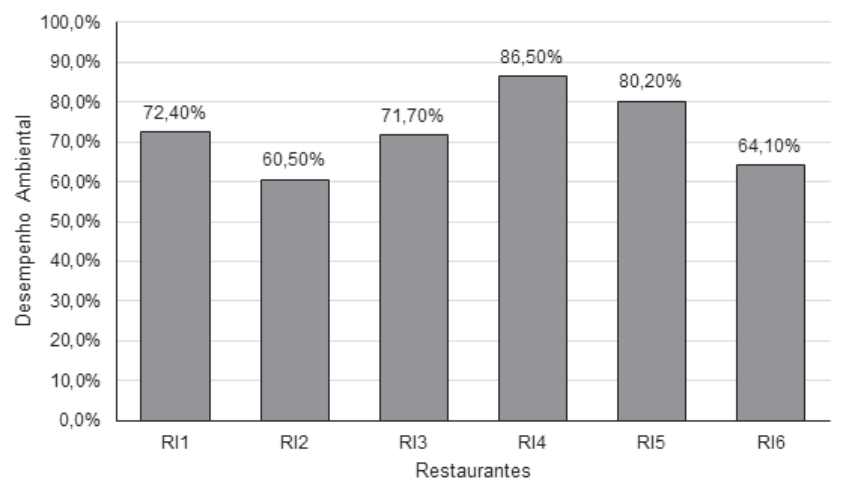

Fonte: Dados da pesquisa.

Os RI 4 e RI 5 apresentaram maior percentual de adequação quanto ao desempenho no uso de recursos naturais, bem como no manejo dos produtos químicos e gestão de resíduo em suas atividades diárias. Ao analisar os cinco parâmetros isoladamente, constatou-se que tanto o consumo de energia elétrica como o de gás, apresentam-se em menores percentuais de adequação nos locais estudados (TABELA 1). Estes achados estiveram relacionados à estrutura física antigas dos prédios e à falta de manutenção preventiva dos equipamentos utilizados no processamento da refeição, que influenciam no desempenho ambiental e consequentemente na eficiência desses recursos.

Tabela 1 - Percentual médio de desempenho ambiental por parâmetros de avaliação em seis restaurantes institucionais de ensino do Rio Grande do Norte, 2019

\begin{tabular}{ccc}
\hline PARÂMETROS & \% Médio & (DP) \\
\hline 1. Água $(n=11)$ & 77,5 & 7,9 \\
2. Energia elétrica $(n=12)$ & 64,7 & 11,7 \\
3. Gás $(n=8)$ & 66,1 & 13,8 \\
4. Produtos Químicos $n=10)$ & 76,7 & 23,6 \\
5. Gestão de resíduos $(n=15)$ & 78,2 & 10,7 \\
\hline
\end{tabular}

$\mathrm{n}$ = quantidade de itens avaliados; $-\%$ = Percentual médio para os seis RI; DP = Desvio Padrão. Fonte: Dados da pesquisa.

$\mathrm{Na}$ avaliação geral do parâmetro água entre os restaurantes, o RI 4 foi o que apresentou o melhor desempenho. Com relação ao parâmetro de gestão resíduos, nas operações de separação e destino dos resíduos sólidos (orgânicos e não orgânicos), o RI 4, apresentou uma gestão diferenciada nesse 
quesito. Os resíduos orgânicos costumam ser destinados para compostagem e/ou suinocultura; e apenas parte é destinada ao aterro sanitário da cidade.

Os resíduos inorgânicos gerados, são destinados inicialmente para uma Unidade de Armazenamento Temporário de Resíduos. Semanalmente, em dois dias distintos, as cooperativas de Coleta Seletiva da Região, fazem o processo de recolhimento desse material e transporta-os para suas sedes, a fim de realizarem o processo de separação dos resíduos - segundo o tipo de material e, posteriormente, efetuar a reciclagem, reutilização e/ou destino ambientalmente adequado.

Por sua vez, no RI 5, no manejo dos resíduos sólidos foi identificado que eram realizados por meio da separação das embalagens recicláveis (papel, plástico, papelão e outros por coleta seletiva); aproveitamento do resto-ingestão dos comensais para compostagem em minhocários; reutilização dos óleos de fritadeira pós-uso, por serviço especializados na fabricação de sabão, ou utilizados para produção de biocombustível em projetos de pesquisas na instituição, caracterizaram-se como atividades diferenciais desempenhadas por esse RI, o que teve implicação nos seus resultados (pontuação de 80,2\% em DA).

Os pontos de controle e monitoramento dos recursos hídricos, energéticos, operacionalização no uso de produtos químicos e no destino final dos resíduos, foram os aspectos gerenciados com maior diligência paralelo às atividades de produção de refeições no RI 4 . 0 menor percentual de classificação foi verificado no RI 2. As condições observadas no RI 2 podem ser justificadas em razão de um fator elencado como fundamental para DA de um serviço: ausência de planejamento para construção de um espaço físico-funcional e sustentável destinado a produção de refeições.

\subsection{CONDICÕ̃ES HIGIENICOSSANITÁRIAS}

No que se refere às condições higienicossanitárias os resultados das análises estão apresentados na Figura 2, conforme o ponto de corte estabelecido para classificar os serviços como "adequado", sendo que os restaurantes 1, 3 e 4 atingiram percentual $\geq 70,0 \%$. Em contrapartida, os RI 2 e 6, foram classificados com avaliação "Ruim”.

Figura 2 - Média de adequação geral para as condições higienicossanitárias nos Restaurantes Institucionais (RI) de ensino do Rio Grande do Norte, 2019

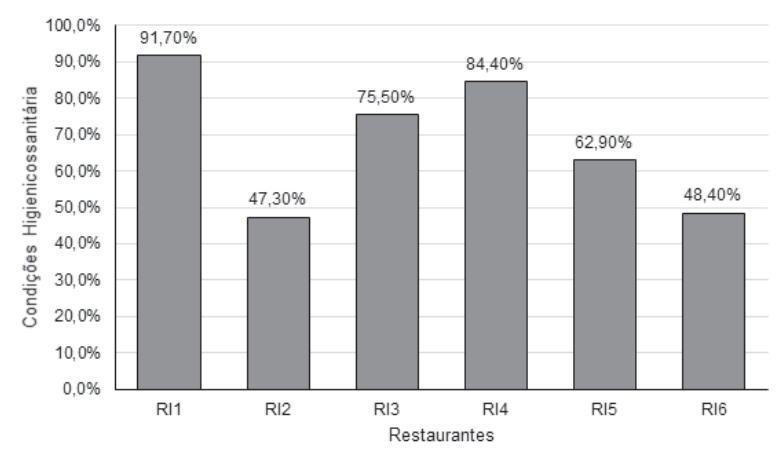

Fonte: Dados da pesquisa. 
Nos resultados apresentados na Tabela 2 pode ser identificado que os melhores desempenhos médios foram obtidos para os blocos que integram, de acordo com a Resolução n 216/2004 da ANVISA, os procedimentos operacionais obrigatórios para serviços de alimentação. No entanto, nas avaliações individuais de cada restaurante, o RI 5 obteve um resultado insatisfatório nesse item $(55,5 \%)$.

Tabela 2 - Percentual de adequação por blocos de avaliação referente às condições higienicossanitárias nos seis Restaurantes Institucionais (RI) de ensino do Rio Grande do Norte, 2019

\begin{tabular}{|c|c|c|}
\hline BLOCOS DE AVALIAÇÃO & Média* & DP \\
\hline Bloco 1 - Edificações, instalações, equipamentos, móveis e utensílios $(n=55)$ & 65,8 & 12,8 \\
\hline Bloco 2 - Higienização instalações, equipamentos, móveis e utensílios ( $n=17$ ) & 77,2 & 24,6 \\
\hline Bloco 3 - Controle integrado de vetores e pragas urbanas $(n=6)$ & 81,4 & 17,0 \\
\hline Bloco 4 - Abastecimento de água $(n=8)$ & 81,6 & 16,1 \\
\hline Bloco 5 - Manejo de resíduos $(n=4)$ & 73,6 & 18,5 \\
\hline Bloco 6 - Manipuladores $(n=13)$ & 68,8 & 16,5 \\
\hline Bloco 7 - Matérias-primas, ingredientes e embalagens ( $n=13$ ) & 73,6 & 27,8 \\
\hline Bloco 8 - Preparação do alimento $(n=24)$ & 66,4 & 22,5 \\
\hline Bloco 9 - Armazenamento e transporte do alimento preparado $(n=6)$ & $68,3^{* *}$ & $44,7^{* *}$ \\
\hline Bloco 10 - Exposição a consumo do alimento preparado $(n=10)$ & 78,6 & 27,2 \\
\hline Bloco 11 - Documentação e registro $(n=24)$ & 54,2 & 34,5 \\
\hline Bloco12 - Responsabilidade $(n=3)$ & 75,9 & 38,7 \\
\hline
\end{tabular}

DP = Desvio Padrão; $\mathrm{n}=$ Números de blocos avaliados conforme se aplicava ao serviço; * Dados coletados em triplicata = intervalo não superior a quinze dias em cada RI; ** Média e DP apenas dos RI 4 e 6 em que o bloco 9 se aplicava.

Fonte: Dados da pesquisa.

Os RI 1, 3, 4 e 5 foram aqueles que obtiveram melhores resultados no bloco de Manejo dos Resíduos da lista de verificação das condições higienicossanitárias (respectivamente 91,6\%; 75,0\%; 75,0\%; $91,8 \%)$. Estes resultados corroboram com aqueles encontrados na avaliação do DA para os mesmos RI no que se refere a "Gestão de Resíduos".

0 bloco relacionado aos manipuladores, apresentou uma média geral preocupante $(68,8 \%)$, contudo os RI 2, 5 e 6 obtiveram resultados ainda mais inferiores, respectivamente 58,9\%, 56,4\% e $48,7 \%$. Esses locais apresentaram em comum situações de não adequação quanto aos requisitos de higienização de mãos no decorrer das atividades, além de ter sido observado o uso de smartphones nas etapas de preparação dos alimentos. Foi identificado nesses três RI problemas relacionados com a disponibilidade de produtos antissépticos e orientações para a realização dessa atividade. 
Em contrapartida, o pior resultado esteve relacionado com a parte de documentação e registro, denotando dessa forma, a fragilidade por parte dos RI em comprovar os processos de avaliação, monitoramento e controle das etapas que possivelmente estejam sendo realizadas ao longo dos serviços.

\section{DISCUSSÃO}

No geral, os resultados da presente pesquisa evidenciam um bom cenário de Desempenho Ambiental por parte dos RI avaliados, considerando o instrumento utilizado. No entanto, quanto ao cumprimento dos requisitos higienicossanitários, os dados apontaram para um panorama que exige maior atenção por parte de alguns restaurantes.

Contatou-se, analisando paralelamente os resultados referentes ao bloco 1 e o percentual médio para os parâmetros ambientais referente a água, energia e gás, que os RI 1, 3 e 4 apresentam-se adequados em ambas as listas de verificação, mostrando que os restaurantes com melhor conservação e uso de suas edificações, instalações, equipamentos, móveis e utensílios apresentam melhor desempenho ambiental. Os RI com os melhores resultados no bloco de Manejo dos Resíduos tiveram desempenho semelhante na "Gestão de Resíduos" da avaliação de DA.

Identificar aspectos ambientais (elemento que podem interagir com o meio ambiente), nas atividades, produtos e serviços e realizar sua determinação, se faz necessário dentro de um serviço de uma Unidade de Alimentação e Nutrição, para que possam ser controlados ou influenciados, e por conseguinte evitar ou reduzir qualquer modificação do meio ambiente, ou seja, resultar no impacto ambiental (ABNT, 2015).

Mistretta e colaboradores (2019) ressaltam que a energia usada no preparo e armazenamento de alimentos (calor e eletricidade), representa uma parte importante do impacto global sobre a demanda de energia, além de repercutir no aquecimento global. A melhoria ambiental dessas fases do ciclo de vida na elaboração de refeições, pode ser facilmente alcançada, consumindo eletricidade produzida por tecnologias de energia renovável e/ou comprando eletrodomésticos de alta eficiência energética.

Estudos dentro da temática de sustentabilidade no campo da Alimentação Coletiva estão em plena ascensão. Vale pontuar que independente do contexto de produção/processamento dos alimentos, seja este um espaço comerciais ou institucionais, adotar práticas sustentáveis em restaurantes, caracteriza-se como uma questão planetária e urgente a ser implementada (BARTHICHOTO et al., 2013; HATJIATHANASSIADOU et al., 2019; STRASBURG; JAHNO, 2017).

Os dados da avaliação higienicossanitária encontrados nos restaurantes estudados, demonstram uma falta de adequação ao cumprimento das boas práticas de manipulação de alimentos, o que pode impactar diretamente na qualidade da alimentação ofertada, afetando a saúde dos usuários. Nos restaurantes que apresentam uma gestão terceirizada (RI 2 e 6) e que não contavam com a presença do nutricionista, foram os que obtiveram os piores resultados, tendo o serviço sido classificado como “ruim". Enquanto nos restaurantes 1, 3 e 4, que são restaurantes de autogestão e que tem participação ativa de nutricionista, percebeu-se uma classificação mais elevada. 
De acordo com a Organização Mundial da Saúde (WHO, 2020), as doenças transmitidas por alimentos (DTA) afetam cerca de 600 milhões de pessoas todos os anos, resultando em aproximadamente 420 mil mortes. Os locais que fornecem alimentos têm impacto direto nessas estatísticas.

Em pleno cenário mundial de pandemia da COVID-19, esses resultados geram inquietações, pois a retomada das atividades em diferentes setores, especificamente nos serviços de alimentação, exige que as medidas básicas de higiene e saúde dos manipuladores e cuidados com superfície inanimadas, sejam reafirmadas e implementadas. Desta forma, cabe aos estabelecimentos públicos ou privados, disponibilizar condições mínimas e seguras para os trabalhadores, além de ficar atento as possíveis reações suspeitas da doença para realizar o afastamento necessário, a fim de evitar contaminação microbiológica e disseminação viral (BEZERRA, 2020; STRASBURG et al., 2020a).

De acordo com a Organização Mundial da Saúde (OMS), em 1989, os manipuladores de alimentos são a principal forma de contaminação dos alimentos que são produzidos em larga escala, portanto, estes desempenham papel importante na segurança dos alimentos em toda a cadeia de produção, desde o recebimento até a distribuição. Em um estudo realizado nos Estados Unidos, chegou-se à conclusão de que $98 \%$ dos surtos alimentares ocorridos em restaurantes, foi por falta de higiene dos funcionários ou por práticas inadequadas na preparação dos alimentos (SHINBAUM et al., 2016).

Um estudo realizado em Vitória/ES avaliou dois serviços de alimentação e nutrição, o item manipuladores, nas duas unidades, ficou com média de $65 \%$ de adequação. As maiores inadequações foram pela não higienização de mãos e uso de roupas pessoais no horário de trabalho (SILVA et al., 2015). Já no estudo de Strasburg e colaboradores (2020b) sobre avaliação de boas práticas com uso de aplicativo móvel em um a rede de restaurantes, o grupo relativo aos manipuladores teve o segundo pior desempenho, atingindo $67,2 \%$ de inadequação, sendo que os problemas mais recorrentes foram relacionados com uniformes, uso de touca, cabelos protegidos e higienização adequada de mãos.

Em relação aos aspectos de registros e documentação, os achados desse estudo corroboraram com os achados de outras pesquisas. Na literatura é comum evidenciar menor conformidade para a categoria Documentação e registo nos serviços de alimentação, como descrito por Nogueira e colaboradores (2019). Essa questão é relevante e merece uma maior atenção por parte das nutricionistas nos serviços de alimentação coletiva, haja vista que esses tópicos costumam ser verificados em inspeções sanitárias e é uma importante ferramenta para a comprovação da garantia de controle de qualidade.

A presente pesquisa sugere uma possível relação entre o Desempenho Ambiental e as Boas Práticas em Serviços de Alimentação, uma vez que os restaurantes com uma boa avaliação de DA tenderam a ser os mesmos que expressaram as condições higienicossanitárias mais satisfatórias, sugerindo que as dimensões ambiental e de saúde podem convergir para a eficiência (otimização dos recursos) e seguridade do produto final servido aos comensais: a refeição. 


\section{CONSIDERAÇÕES FINAIS}

O desempenho ambiental dos restaurantes avaliados foi considerado bom numa avaliação geral. No entanto o consumo de energia elétrica e de gás, apresentaram os menores percentuais de adequação nos locais estudados possivelmente devido ao fato da maioria das instalações avaliadas serem estruturas antigas.

Os resultados da presente pesquisa mostraram, também, que metade dos IR de ensino avaliados apresentaram falta de conformidade com alguns aspectos relacionados na legislação sanitária vigente. 0 item documentação e registro foi o que se apresentou em maior desacordo com as boas práticas de manipulação.

A realização de avaliações e monitoramento das condições ambientais e higienicossanitárias é fundamental para os serviços de alimentação para coletividade, especialmente no contexto atual do mundo em que vivemos. Dessa forma, utilizar instrumentos de avaliação e, principalmente, utilizá-los como recursos para a melhoria dos serviços é imprescindível.

\section{REFERÊNCIAS}

ABERC. Associação Brasileira de Empresas de Refeições Coletivas. Mercado Real. Disponível em: https://www.aberc.com.br/mercadoreal.asp?IDMenu=21. Acesso em: 25 mar. 2021.

ABNT. Associação Brasileira de Normas Técnicas. NBR ISO 14001: Sistemas de gestão ambiental Requisitos com orientações para uso. ABNT: Rio de Janeiro, 2015.

BARTHICHOTO, M. et al. Responsabilidade Ambiental: perfil das práticas de sustentabilidade desenvolvidas em unidades produtoras de refeições do bairro de Higienópolis, Município de São Paulo. Qualit@s, v.14, n. 1, p. 78-95, 2013.

BEZERRA, I. N. Away-from-home food during coronavirus pandemic. Int J Env Res Pub He, v. 23, n. 10, p. 1855, 2020.

BRASIL. Ministério da Saúde. Agência Nacional de Vigilância Sanitária. Resolução RDC no 216, de 15 de setembro de 2004. Dispõe sobre Regulamento Técnico de Boas Práticas para Serviços de Alimentação. Brasília, DF: ANVISA. 2004. Disponível em: http://antigo.anvisa.gov.br/ documents/10181/2718376/RDC_216_2004_COMP.pdf/66f5716e-596c-4b9d-b759-72ce49e34da0. Acesso em: 25 mar. 2021

BRASIL. Ministério da Saúde. Agência Nacional de Vigilância Sanitária. Guia de boas práticas nutricionais: Restaurantes Coletivos. Brasília, DF: ANVISA. 2014. Disponível em: http://www6.ensp. 
fiocruz.br/visa/files/Guia+de+Boas+Pr\%C3\%A1ticas+Nutricionais+para+Restaurantes+Coletivos. pdf. Acesso em 25 mar. 2021.

CFN - Conselho Federal de Nutricionistas. Resolução CFN nº 600, de 25 de fevereiro de 2018. Dispõe sobre a definição das áreas de atuação do nutricionista e suas atribuições, indica parâmetros numéricos mínimos de referência, por área de atuação, para a efetividade dos serviços prestados à sociedade. Brasília, DF: CFN. 2018. Disponível em: https://www.cfn.org.br/wp-content/uploads/ resolucoes/Res_600_2018.htm. Acesso em: 25 mar. 2021

CFN. Conselho Federal de Nutricionistas. Recomendações do CFN: boas práticas para atuação do nutricionista e do técnico em nutrição e dietética durante a pandemia de coronavírus. boas práticas para atuação do nutricionista e do técnico em nutrição e dietética durante a pandemia de coronavírus. Brasília, DF: CFN. 2020. Disponível em: https://www.cfn.org.br/wp-content/ uploads/2020/03/nota_coronavirus_3-1.pdf. Acesso em: 25 mar. 2021.

HARMON, A. H. et al. Position of the American Dietetic Association: food and nutrition professionals can implement practices to conserve natural resources and support ecological sustainability. J Am Diet Assoc, v. 107, n. 6, p. 1033-1043, jun. 2007.

HATJIATHANASSIADOU, M. et al. Environmental Impacts of University Restaurant Menus: A Case Study in Brazil. Sustainability, v. 11, n. 19, p. 5157, 20 set. 2019.

MISTRETTA, M. et al. Energy and environmental life cycle assessment of an institutional catering service: An Italian case study. Sci Total Environ, v. 657, 2019.

NOGUEIRA, J. P. et al. Food handling conditions of university canteens. Rev Chil Nutr, v. 46, n. 5, p. 527-534, out. 2019.

NOGUEIRA, J. P. et al. Sustainable perspective in public educational institutions restaurants: from foodstuffs purchase to meal offer. Sustainability, v. 12, n. 11, p. 4340, 26 maio 2020.

OMS - Organización Mundial de la Salud. Métodos de vigilancia sanitaria y de gestión para manipuladores de alimentos: informe de una reunión de consulta de la OMS. Ginebra: OMS, 1989. Disponivel em: https://apps.who.int/iris/bitstream/handle/10665/38647/WHO_TRS_785_spa. pdf?sequence=1\&isAllowed=y. Acesso em: 25 mar. 2021.

SACCOL, A. L. F. et al. Instrumentos de apoio para implantação das boas práticas em empresas alimentícias. Rio de Janeiro: Rubio, 2012. 
SANTOS, M. V. et al. Os Restaurantes por peso no contexto de alimentação saudável fora de casa. Rev Nutr, v. 24, n. 4, p. 641-649, 2011.

SHINBAUM, S. et al. Evaluating your obligations for employee training according to the Food Safety Modernization Act Food Control, v. 60, p. 12-17, 2016.

SILVA JÚNIOR, E. A. Manual de controle higiênico-sanitário em alimentos. São Paulo: Editora Varela, 2007.

SILVA, L. C. et al. Boas práticas na manipulação de alimentos em unidades de alimentação e nutrição. Demetra, v. 10, n. 4, p. 797-820, 2015.

STRASBURG, V. J. Desenvolvimento de instrumentos para a avaliação de desempenho ambiental na produção de refeições. 2016. 142 f. Tese (Doutorado em Qualidade Ambiental) - Universidade Feevale, Novo Hamburgo, 2016.

STRASBURG, V. J. et al. COVID-19: Contingency actions for the provision of meals to employees of a university hospital in southern Brazil. Sci Med, v. 30, n. 1, p. e38769, 2020 a.

STRASBURG, V. J. et al. Diagnóstico de boas práticas por meio de uso de aplicativo móvel: estudo de caso em galeterias no Sul do Brasil. Discipl Sci Ciên Saúde, v. 21, n. 1, p. 171-185, 2020 b.

STRASBURG, V. J.; JAHNO, V. D. Application of eco-efficiency in the assessment of raw materials consumed by university restaurants in Brazil: A case study. J Clean Prod, v. 161, p. 178-187, 10 set. 2017.

UN. United Nations. Transforming our world: the 2030 Agenda for Sustainable Development. New York: UN. 2015 Disponível em: https://sustainabledevelopment.un.org/content/ documents/21252030 Agenda for Sustainable Development web.pdf. Acesso em: 25 mar. 2021.

VON KOERBER, K. et al. Wholesome Nutrition: an example for a sustainable diet. Proc Nutr Soc, v. 76, n. 1, p. 34-41, 2017.

WALLS, H. et al. Food security, food safety \& healthy nutrition: are they compatible? Global Food Sec, v. 21, n. April, p. 69-71, 2019.

WHO - World Health Organization. Food safety. Disponível em: https://www.who.int/health-topics/ food-safety. Acesso em: 25 mar. 2021. 
1 Mestre em Nutrição. Universidade Federal do Rio Grande do Norte - UFRN); Nutricionista.

E-mail: josinogueiira147@gmail.com

2 Mestranda em Nutrição, Universidade Federal do Rio Grande do Norte - UFRN. Nutricionista.

E-mail: mariahatji@hotmail.com

3 Doutor em Qualidade Ambiental; Nutricionista; Professor Adjunto IV do Departamento de Nutrição, Universidade Federal do Rio Grande do Sul - UFRGS.

E-mail: virgilio_nut@ufrgs.br

4 Doutora em Biotecnologia; Nutricionista; Professora Adjunto classe $C$ do Departamento de Nutrição, Universidade Federal do Rio Grande do Norte - (UFRN.

E-mail: priscillanutri@hotmail.com

5 Nutricionista; Professora Adjunto IV do Departamento de Nutrição, Universidade Federal do Rio Grande do Norte UFRN. E-mail: larissaseabra@yahoo.com.br

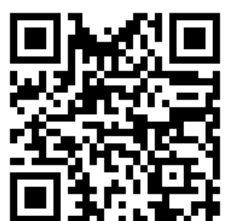

A autenticidade desse artigo pode ser conferida no site https://periodicos. set.edu.br

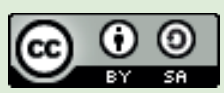

Este artigo é licenciado na modalidade acesso abertosob a Atribuição-Compartilhalgual CC BY-SA

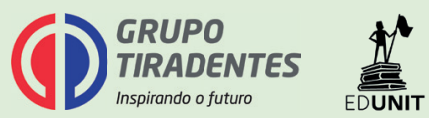

